

\title{
POTENCIAL DE DEMANDA ENERGÉTICA CERO EN ENVOLVENTES INDUSTRIALES: EL CASO DE LA VINIFICACIÓN EN ZONAS DE GRAN AMPLITUD TÉRMICA
}

\author{
THE POTENTIAL OF ZERO ENERGY DEMAND IN INDUSTRIAL \\ ENVELOPES: \\ THE CASE OF VINIFICATION IN AREAS OF GREAT THERMAL \\ AMPLITUDE
}

\author{
ALBA INÉS RAMOS SANZ \\ Universidad Nacional de San Juan (UNSJ)-Consejo Nacional de \\ Investigaciones Científicas y Tecnológicas (CONICET) \\ San Juan Argentina \\ draarqalbaramossanz@gmail.com
}

\section{RESUMEN}

En el estudio presentado en este artículo se desarrolla un modelo matemático para la simulación en tiempo real de un envolvente de vinificación representativo de la tecnología de vinificación nacional -de hormigón armado-, y se lleva a cabo un seguimiento térmico tanto del vino almacenado en este interior como en uno de acero inoxidable, con el empleo de sensores de temperatura HOBO U-12. Mediante tal simulación, se contempla el efecto producido sobre la estabilidad térmica del vino y la demanda térmica originada en la convección forzada -causada por las brisas-, bajo diversas

configuraciones de bodegas. Con la herramienta validada, se realiza una sencilla optimización pasiva sobre el caso representativo. Finalmente, comparando los resultados de los casos simulados

optimizados y de los casos reales, se concluye una mayor eficiencia termo-energética del caso representativo, frente a los de acero inoxidable. Asimismo, se observa que la estabilidad térmica del vino con demanda cero es factible a partir del empleo de envolventes adecuadas al clima analizado; con sencillas medidas de optimización, dicha estabilidad se acerca al prototipo de demanda cero perseguida en los modelos de referencia para la gestión de la energía

\section{Palabras clave}

gestión de la energía, calidad del producto, vinificación, modelización térmica

\section{ABSTRACT}

In this study, a mathematical model was developed for the real-time simulation of a winemaking envelope representative of the national vinification technology of reinforced concrete. Thermal monitoring of the wine stored inside this envelope and another of stainless steel was carried out using HOBO U-12 temperature sensors. The simulation studied the effect on the thermal stability of the wine and the thermal demand originating in the forced convection caused by breezes, under various cellar configurations. With the validated tool, a simple passive optimization was performed on the representative case. Finally, comparing the results of the optimized, simulated cases and the real cases, it was concluded that the representative case has a higher thermo-energy efficiency compared to the stainless-steel cases. Likewise, it was observed that the thermal stability of the wine with zero

demand is feasible through the use of envelopes that are suitable for the climate analyzed; with simple optimization measures, this stability comes close to the zero-demand prototype sought after in reference models for energy management 


\section{INTRODUCCIÓN}

El potencial de ahorro de energía en la República Argentina señala que este podría representar un $45 \%$ en el sector residencial, un $28 \%$ en el sector de transporte, un $20 \%$ en el sector industrial y un $7 \%$ en el sector público. En el sector industrial, particularmente, el potencial de ahorro energético se encuentra señalado en los siguientes focos:

1. Sistemas de gestión de la energía (SGEn).

2. Diagnósticos energéticos.

3. Motores eficientes y variadores de velocidad.

4. Eficiencia en calderas industriales.

Los programas vigentes en el país enmarcados en eficiencia energética están orientados a dos áreas: el Etiquetado de Eficiencia Energética y la Eficiencia Energética en la Industria, mediante la adaptación de la Norma IRAM/ISO 50001 (2016). Estas iniciativas buscan generar una necesidad de eficiencia en la producción industrial, apalancada por los altos costos de la energía. La norma IRAM/ISO 50001 hace hincapié en el foco 1 (SGEn) señalado en el párrafo anterior y conforma, hasta el momento, el marco regulatorio que se le otorga a nivel nacional al abordaje del problema de la demanda energética en la industria.

El reconocimiento de la importancia de la temperatura de control de los procesos en la industria agroalimentaria demanda ingentes inversiones sobre los recursos tecnológicos, económicos y energéticos en procesos y control de calidad. La gestión de la energía aplicada al sector industrial impacta negativamente sobre la sustentabilidad energética y rentabilidad del sector económico. Parte del objetivo de mejorar la performance energética de una industria alimentaria a través de una correcta gestión de la energía, lo constituye -en el caso de una bodega- la apropiada selección tecnológica de las envolventes de vinificación. Trabajos anteriores basados en datos registrados con sensores y procesados estadísticamente demuestran la existencia de correlación empírica en función de la envolvente de los fermentadores, entre la temperatura del vino en el interior de los tanques de vinificación y la temperatura del aire en el exterior de la bodega (Ramos Sanz, 2014). En ese marco, se llevaron a cabo estudios experimentales que registraron temperaturas de conservación del vino más elevadas que las recomendadas por los especialistas. Los resultados estadísticos indicaron, para verano, una temperatura de $+4 \mathrm{~K}$ sobre el margen superior de conservación de vinos comunes y, en invierno, una media mínima el doble de cálida de lo que señala la teoría. La desviación estándar en ambos casos fue también superior en un $30 \%$ a la teórica, admitiendo un $+0,4 \mathrm{~K}$ de inestabilidad térmica.

Una selección inteligente de las envolventes para la gestión de la energía que se orienta hacia el potencial pasivo de la industria, podría generar ahorros de más del $50 \%$ de energía en vinificación. Esta definición de la demanda energética en función de capital físico atiende al mismo fin que la premisa de los Net Zero Buildings (NZEB), persiguiendo un máximo confort (del producto) con un mínimo empleo de recursos energéticos. El desconocimiento de la temperatura del producto y su demanda energética perjudica la gestión de la energía en la industria, no siendo posible determinar una acción de mejora. Esta información es la base para la gestión tanto de la calidad del producto como de la eficiencia energética en el sector de procesos de la industria.

Por consiguiente, se considera importante disponer de una herramienta de simulación capaz de pre-diagnosticar cuantitativamente las demandas termo-energéticas y la optimización mediante estrategias pasivas. En la actualidad, se registran escasos datos sobre la optimización pasiva de las envolventes industriales en general, en los cuales se contemple la variedad tipológica y la diversidad material y morfológica de los casos analizados. La cuantificación del efecto de las medidas pasivas de optimización de la envolvente de vinificación (EV) proporciona información relevante. Previamente (Ramos Sanz, A: 2015), se difundió un modelo para simular el potencial de optimización de las envolventes de vinificación mediante estrategias bioclimáticas pasivas, con el fin de reducir la demanda energética en control térmico. Se determinó, entonces, el porcentaje de las cargas de frío que se originan en el intercambio de la EV con el medio climático, sobrecargando el sistema de refrigeración. Las estrategias pasivas -optimizaciones- experimentadas para período cálido y sus resultados se enumeran a continuación;

1. Empleo de envolventes subterráneas (79,5\%)

2. Uso de envolventes apiladas en su eje vertical $(58,6 \%)$

3. Incorporación de aislación térmica exterior a la superficie de la EV (50,5\%)

4. Aplicación de terminaciones de acabado claro en las superficies expuestas a la radiación solar directa (51\%)

5. Utilización de envolventes expuestas a la intemperie para promover el recurso de la radiación nocturna $(71,5 \%)$ desde la cubierta hacia el cielo.

Entre los resultados de las optimizaciones energéticas mediante la aplicación de las estrategias pasivas, el valor medio obtenido fue del $47,7 \%$ de reducción de la carga en control térmico. Entre las variables no experimentadas se encuentra el impacto del viento sobre el intercambio térmico de los fermentadores con el entorno, según su forma y su distribución en el solar. Asimismo, estos resultados corresponden a modelos de simulación simplificados, en régimen estacionario.

En las economías vitivinícolas del mundo como el Reino Unido, Australia y Estados Unidos, se promueve la búsqueda de soluciones a las elevadas demandas energéticas de las bodegas. Best Winery (California Energy Commission; 2005), British Columbia Sustainable Winegrowing Program (2011; UK) y Winery Energy Saving Toolkit (Australian Wine Research Institute, 2012;) son algunas herramientas 
desarrolladas por dichos países. Mientras que los resultados cuantitativos normalizados de la aplicación de estrategias pasivas son conocidos en casos de viviendas y edificios residenciales, comerciales y administrativos, son escasos los estudios sistemáticos sobre estos potenciales aplicados a la industria del vino. A pesar de los antecedentes citados, no se tiene conocimiento de experimentación termoenergética en modelos de envolventes de vinificación y su impacto en la demanda energética.

\section{METODOLOGÍA}

\section{CONTEXTO DE LA ZONA DE ESTUDIO.}

Contexto geográfico

La zona de estudio comprende el Valle del Tulum, ubicado en la ciudad de San Juan (República Argentina), cuyo clima es, de entre los climas cálidos secos de América del Sur, el más extremo. Se localiza, concretamente, a $600 \mathrm{msnm}$, en los $30^{\circ} 52^{\prime}$ de Latitud Sur y $68^{\circ} 59^{\prime}$ de Longitud en dirección Oeste. Como en otras regiones vitivinícolas del mundo, la marcada amplitud térmica del clima continental agudiza la dependencia energética de la vinificación, originada en el control térmico de procesos. Este tipo de clima se caracteriza por un terroir que se repite, por ejemplo, en la zona vitivinícola de la Sierra Foothills ${ }^{1}$. El Valle del Tulum es, en efecto, una zona cálida, con amplitudes térmicas diarias superiores a los +/- 14 K (IRAM11603, 1996). A lo largo del año, los vientos son de dirección predominantemente Sur. La velocidad de los mismos se duplica en verano, alcanzando una media de $13 \mathrm{Km} / \mathrm{h}$ (IRAM11603, 1996). En dicha estación, se registra una irradiancia solar media de $850 \mathrm{~W} / \mathrm{m}^{2}$ en horas del mediodía y los aportes solares son significativos sobre el plano horizontal y este-oeste. En invierno, las superficies orientadas al norte reciben los rayos directos durante más horas y mayor intensidad que las demás orientaciones. Es necesario aclarar que las condiciones climáticas durante el relevamiento no son representativas del clima considerado, son de hecho una excepción. Esto se debe a la dificultad de coordinar con la empresa los períodos de análisis. Sin embargo, los datos recogidos son útiles a los efectos de disponer de datos reales de temperatura.

\section{Contexto energético nacional}

En este estudio se aborda la demanda energética en términos de energía eléctrica destinada al funcionamiento de los sistemas de control térmico de los tanques de vinificación. Esto se basa en la mayor tecnificación de los procesos y su creciente dependencia energética industrial. La República Argentina se encuentra en un momento de transición energética; desde 2016 en adelante los costos de la energía eléctrica se han incrementado un 182\%

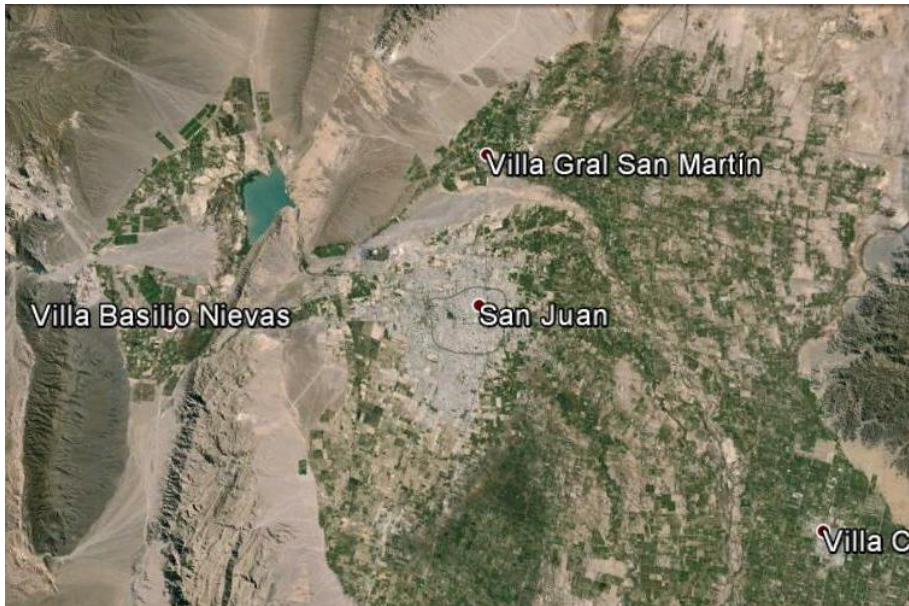

Figura 1. Imagen satelital del Valle del Tulum, Ullum y Zonda, en la Provincia de San Juan. Fuente: Recursos propios, mediante herramienta Google earth.

para Pequeñas y Medianas Empresas (PyMEs) a causa de una política fiscal contractiva. Este incremento afecta los Índices de Precios al Consumidor (IPC) que han variado un $83 \%$ desde 2016 hasta la actualidad, lo cual señala la incidencia del incremento tarifario sobre el nivel de precios del país (Observatorio Económico Social UNR, 2017). Sin embargo, en Argentina la aplicación de las normativas de Eficiencia Energética en la Industria no es obligatoria. Su adhesión es voluntaria y la misma no percibe ningún incentivo o beneficio por parte del Estado o de las entidades bancarias. En este panorama, se pueden hallar los motivos de este tipo de PyME para no integrar acciones de Responsabilidad Social Empresaria (RSE) a sus procesos, con la finalidad de obtener una disminución de la demanda energética y un incremento de su rentabilidad, en claro perjuicio de su sostenibilidad.

\section{SELECCIÓN DEL CASO TESTIGO E INSTRUMENTAL UTILIZADO}

Entre las EV, existe cierta diversidad. Éstas pueden ser distinguidas por su ubicación: si son sobre-rasantes, se habla de tanques -acero o metal- o piletas -hormigón o mampostería- y, si son subterráneas, se habla de cisternas -hormigón o mampostería-. El caso seleccionado para la modelización es altamente representativo de las industrias vitivinícolas del país, pues alcanza el $68 \%$ de la población provincial de EV (Instituto Nacional de Vitivinicultura INV, 2008). El mismo se compone de un volumen cilíndrico de hormigón armado ( $e=0,27 \mathrm{~m}$ ), cuyo acabado interior es de pintura epoxídica y en el exterior se conserva el hormigón a la vista. La capacidad del caso testigo seleccionado

[1] Las temperaturas medias máximas de verano $\left(\mathrm{TMX} ; 33^{\circ} \mathrm{C}\right)$ y las temperaturas medias mínimas de invierno $\left(\mathrm{TMN} ; \approx 12^{\circ} \mathrm{C}\right.$ ) entre ambas localidades vitivinícolas son similares, siendo Sacramento un poco más cálido en invierno debido a su mayor humedad ambiental. 
es aproximada a la capacidad promedio del total de la población de EV registrada en el país, con un volumen de $90000 \mathrm{~L}$ de vino almacenado. El muro curvo y la cubierta de la EV se encuentran en intercambio directo con el ambiente exterior, mientras la base de la misma (entrepiso de losa) y un muro recto está en contacto con el vino de la pileta subyacente (inferior y lateral) al caso analizado. En la configuración de la bodega, esta unidad se encuentra dispuesta en conjunto con otras EV -como se observa en la Figura 2-, cual banco de tubos;

Así, se relevan los datos de temperatura interior y exterior con sensores HOBO U-12 cuya precisión es de $\pm 0,5^{\circ} \mathrm{C}$ a $20{ }^{\circ} \mathrm{C}$; y un termómetro común, instrumental del enólogo. El efecto de la radiación solar difusa está implícito en los valores registrados de temperatura del aire y de temperatura superficial exterior. Con el fin de analizar la transferencia de calor sin las complejidades de los modelos cinéticos -que implican complejos procesos químicos-, se selecciona el período correspondiente al estado de conservación del vino, durante el cual no se manifiestan reacciones químicas significativas. Este límite impuesto resulta eficaz para determinar la demanda energética base de un tipo de EV. Luego, a esa demanda es posible adicionarle las necesidades de frío originadas en la fermentación, en base a datos específicos tales como la temperatura de ingreso de la uva y el grado de azúcar contenido en el grano. La misma se determinada por una sencilla fórmula conocida por los enólogos (véase Oreglia; 1978).

\section{DESARROLLO DEL MODELO MATEMÁTICO}

El modelo matemático aquí empleado se construye considerando que el flujo de calor es unidireccional y radial. Las condiciones de frontera son la temperatura exterior y la temperatura en el nodo central, trasponiéndose los resultados por simetría desde el centro de la EV, hacia la otra mitad geométrica. En el exterior de la vasija, circulan las brisas provenientes del sur-este, a una velocidad media -considerada constante- de $13 \mathrm{~km} / \mathrm{h}$. Las EV se encuentran ubicadas a la intemperie, dada la ingente capacidad de almacenamiento. La distribución frecuente de estos elementos es semejante a la de un banco de tubos, sobre el cual impactan las brisas en diagonal a la impronta de la bodega. Se consideran dos situaciones donde el viento afecta la transferencia de calor, según la localización de la EV: la primera contempla la vasija como el primer elemento sobre el cual impacta el viento a la velocidad original; la segunda considera a la EV protegida por otras, por entremedio de las cuales circula el fluido, variando su velocidad. El desplazamiento del fluido en ambas situaciones se desarrolla de forma turbulenta.

Los valores de las conductancias superficiales interiores $\left(h_{\mathrm{i}}\right)$ y exteriores $\left(h_{e}\right)$ se consideran constantes. Ello, debido al leve gradiente térmico y la gran inercia térmica del producto en conservación, en el caso de $h_{i}$, y porque depende fuertemente de otros factores diferentes a la variación térmica $\Delta T$, en el caso de $h$. A continuación, se procede al cálculo en diferencias finitas mediante el método explícito. Los valores de las condiciones iniciales $\left(T_{0}^{i} T_{0}^{i}\right.$ y $\left.T_{4}^{i} T_{4}^{i}\right)$ se estiman a partir de los datos experimentales de temperatura exterior $(T)$ e interior $\left(T_{i}\right)$ recogidos con sensores $\mathrm{HOBO} U-12$ a cada minuto

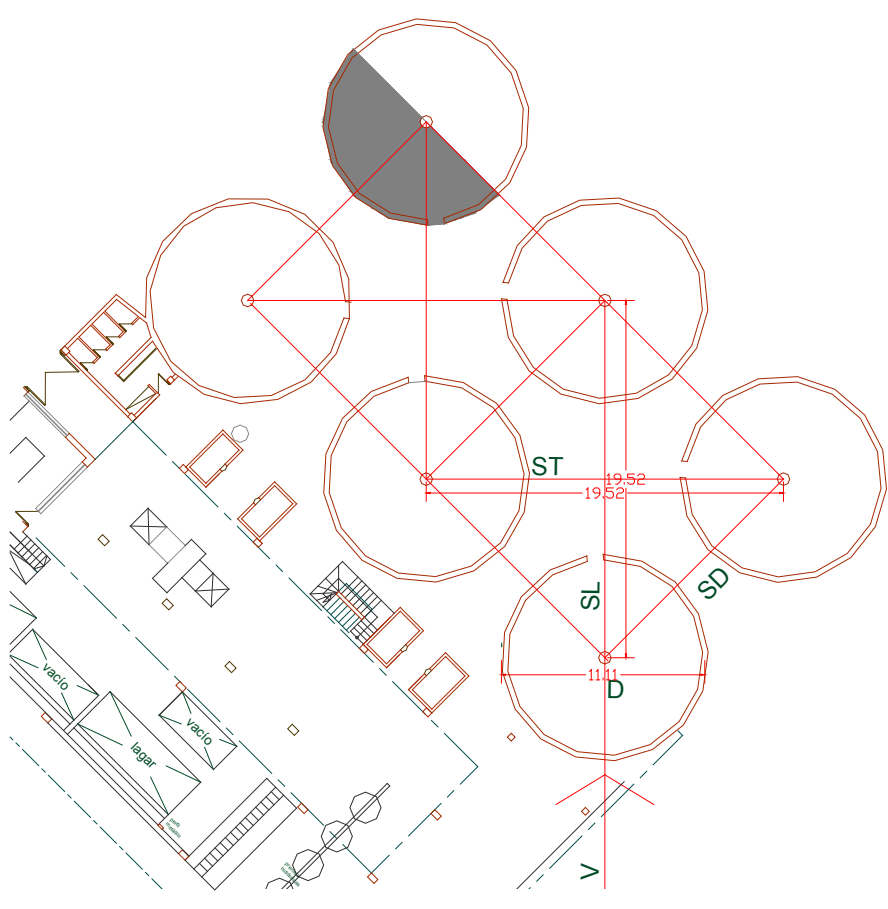

Figura 2. Planta de la distribución de las EV en conjuntos alineados. Fuente: Fotografía tomada por la autora durante relevamiento.

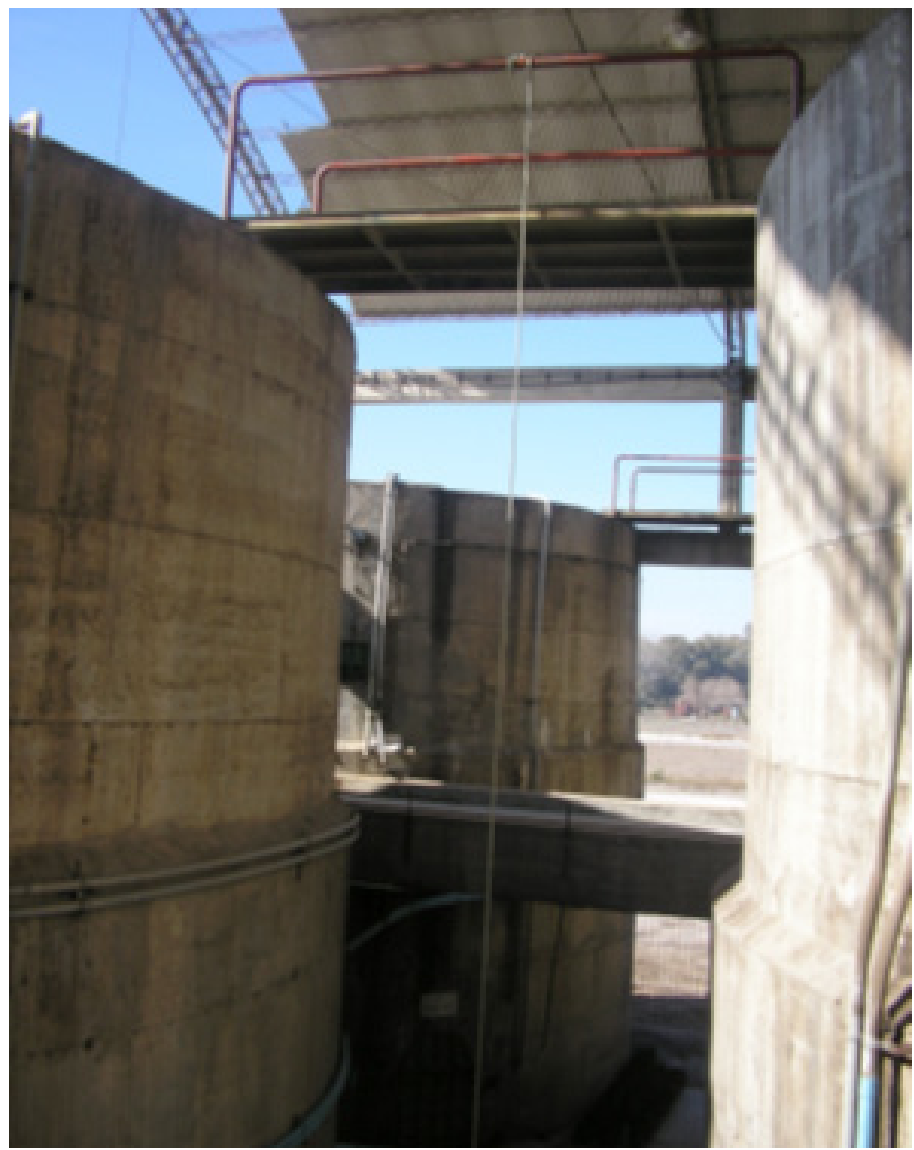

Figura 3. Imagen de la envolvente de las EV. Fuente: Elaboración de la autora en función de datos reales. 
y complementando los datos semi-experimentales de $h_{i}$ y $h_{e}$. Para proceder al empleo de este método, se deben discretizar las envolventes, en unidades diferenciales. En la práctica, se suelen tomar tamaños de discretización $(\Delta x \Delta x)$ entre 0.05 y 0.10 $\mathrm{m}$. Estas dimensiones reducen el error de truncamiento que se arrastra en las ecuaciones consecutivas, a medida que pasa el tiempo. Para la envolvente-tipo definida, se consideran 5 nodos $(M)$;

$$
\Delta x=\frac{L}{M-1}=\frac{0,27 m}{5-1}=0,0675 \mathrm{~m} \quad(\mathrm{~m})(1)
$$

Con este espaciamiento nodal se determina el número de malla de Fourier en función del espaciamiento temporal $(\Delta \mathrm{t})$ requerido. Dado que los datos recogidos con sensores de temperatura se programaron cada un minuto, entonces se define $\Delta t=60$ segundos:

$$
\tau=\frac{\alpha \Delta t}{\Delta x^{2}}=0,014
$$

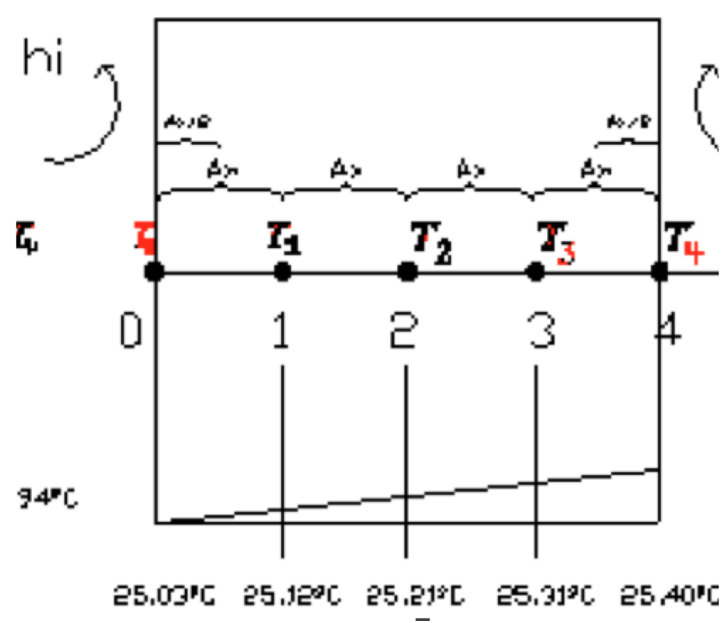

Figura 4. Discretización del elemento pared, en cinco nodos desde $T_{0}^{\mathrm{i}} T_{0}^{\mathrm{i}}$ hasta $T_{4}^{\mathrm{i}} T_{4}^{\mathrm{i}}$. Fuente: Elaboración de la autora.

\begin{tabular}{|l|l|l|}
\multicolumn{1}{c|}{$\begin{array}{c}\text { Conductancias } \\
\text { exteriores } \\
{[\mathrm{Wh} / \mathrm{m} 2 \mathrm{~K}]}\end{array}$} & $\begin{array}{c}\text { Configuración } \\
\text { EV aislada }\end{array}$ & $\begin{array}{c}\text { Configuración } \\
\text { EV en conjunto }\end{array}$ \\
\hline $\mathrm{h}_{\text {laminar medio }}$ & 1,775 & 1,834 \\
\hline $\mathrm{h}_{\text {turbulento medio }}$ & 24,957 & 31,653 \\
\hline $\mathrm{h}_{\mathrm{e}(\text { Lesino) }}$ & 18,553 & 19,422 \\
\hline $\mathrm{h}_{\mathrm{e}(\text { Colombié) }}$ & 6,337 & 6,557 \\
\hline
\end{tabular}

Tabla 1. Valores semi-experimentales obtenidos y valores de referencia de la bibliografía consultada, en relación a las conductancias superficiales exteriores, para el intercambio térmico por convección forzada de las brisas sobre las envolventes ubicadas en unidades aisladas y agrupadas. Fuente: Elaboración propia
Nuevamente, se expresan las ecuaciones diferenciales, en este caso para los nodos interiores, $\mathrm{M}_{\mathrm{n}+1}$;

$$
\lambda A \frac{\left(T_{0}^{\mathrm{l}}-T_{1}^{\mathrm{l}}\right)}{\Delta x}+\lambda A \frac{\left(T_{2}^{\mathrm{l}}-T_{1}^{\mathrm{l}}\right)}{\Delta x}=\rho A \Delta x c_{p} \frac{T_{1}^{\mathrm{l}+1}-T_{1}^{\mathrm{l}}}{\Delta t}
$$

Para los nodos frontera $\mathrm{M}_{\mathrm{Tsi}}$ y $\mathrm{M}_{\mathrm{Tse}}$;

$$
\begin{aligned}
& \lambda A \frac{\left(T_{n}^{\mathrm{l}}-T_{0}^{\mathrm{l}}\right)}{\Delta x}+h A\left(T_{\text {vino }}^{\mathrm{i}}-T_{0}^{\mathrm{i}}\right)=\rho A \frac{\Delta x}{2} c_{p} \frac{T_{0}^{\mathrm{l}+1}-T_{0}^{\mathrm{l}}}{\Delta t} \\
& \lambda A \frac{\left(T_{n}^{\mathrm{l}}-T_{n+1}^{\mathrm{l}}\right)}{\Delta x}+h A\left(T_{\infty}^{\mathrm{i}}-T_{n+1}^{\mathrm{i}}\right)=\rho A \frac{\Delta x}{2} c_{p} \frac{T_{n+1}^{\mathrm{l}+1}-T_{n+1}^{\mathrm{l}}}{\Delta t}
\end{aligned}
$$

El criterio de estabilidad requerido para el método explícito para los valores $\Delta \mathrm{t}$ y $\Delta \mathrm{x}$ definidos indica que el número adimensional de malla de Fourier 0 debe ser $\geq 0$. Este criterio se define con el valor del coeficiente más pequeño de las funciones $T_{n}^{1+n} T_{n}^{1+n}$ en las condiciones más restrictivas que son los nodos frontera, en este caso el valor de $h_{i}$. Si este criterio no es verificado, los resultados de la simulación pierden coherencia y el modelo pierde su confiabilidad absolutamente.

\section{VALIDACIÓN DE LA HERRAMIENTA DE SIMULACIÓN}

Mediante la herramienta de simulación, se obtiene el perfil de temperaturas del vino en conservación en tiempo real, almacenado dentro de una EV inserta en una configuración tipo conjunto de unidades. Se observa que luego de 250 pasos temporales de 60 segundos -equivalentes a 4 horas- el vino comienza a elevar su contenido de energía interna, perdiendo su estabilidad térmica. La parte del fluido más cercana a los muros, amortigua el impacto térmico del clima exterior con sacrificio de la homogeneidad térmica del volumen de vino. La Figura 5 representa el comportamiento del vino almacenado, a lo largo de un período breve de 6 días. Cada línea de color indica un nodo espacial, proyectado en el paso temporal de 60 segundos. En este período, la temperatura interior del vino ha oscilado fuertemente, acompañando las variaciones climáticas exteriores. Los datos del sensor en la masa del vino señalan el comportamiento térmico de la capa límite del fluido, ya que en ese punto se advierten variaciones térmicas de manera semejante y paralela a los nodos más próximos al límite del sistema. El error relativo entre la información registrada y la provista por la simulación, alcanza el 10\% para 306 datos recogidos. Este margen implica los saltos térmicos registrados por los sensores, los que se deben a causas externas que motivan out-layers dentro de la normalidad de los datos, considerándose, en consecuencia, un error relativo menor. En este tipo de métodos iteractivos en los cuales una función se encadena a la anterior y a la siguiente, a la superior y a la posterior -en un esquema matricial-, se promueve además un error de tipo acumulativo que crece con la cantidad de pasos temporales y espaciales que requiera el modelo. 


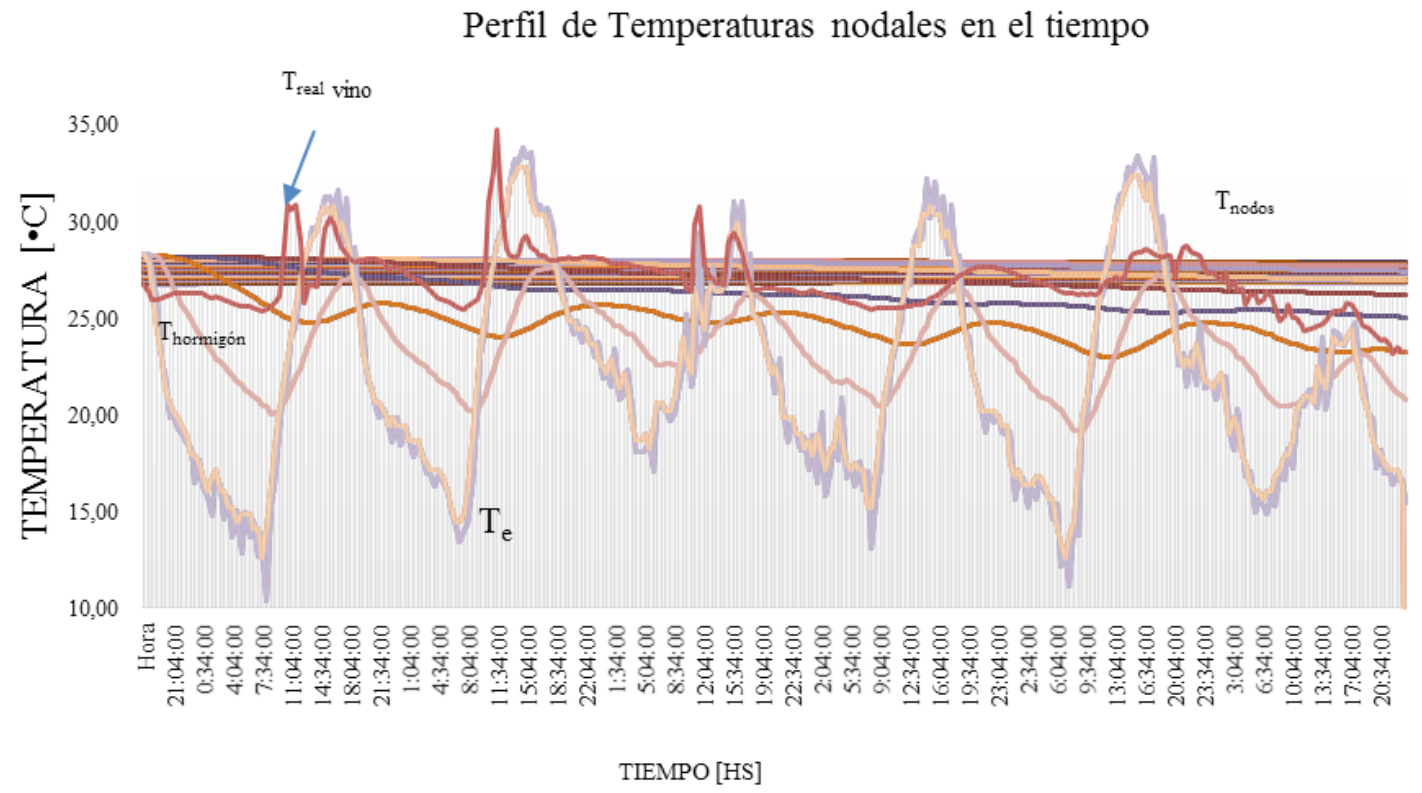

Figura 5. Perfil de temperaturas de los diferentes nodos espaciales a lo largo de seis días. Fuente: Elaboración de la autora en base a modelado con herramienta Excell.

\begin{tabular}{|c|c|c|c|c|c|c|c|c|c|c|c|}
\hline \multicolumn{3}{|c|}{ Propiedades del Aire a $25^{\circ} \mathrm{C}$} & \multicolumn{3}{|c|}{ EV AISLADA O PRIMERA } & \multicolumn{3}{|c|}{ EV EN CONJUNTO ESCALONADO } & \multicolumn{3}{|c|}{ EV EN CONJUNTO ALINEADOS } \\
\hline Densidad & $(\mathrm{kg} / \mathrm{m} 3)$ & 1,2 & Vel Viento & $(\mathrm{Km} / \mathrm{h})$ & 13,000 & Vel Viento & $(\mathrm{Km} / \mathrm{h})$ & 12,2 & Vel Viento & $(\mathrm{Km} / \mathrm{h})$ & 31,3 \\
\hline Calor Espe & $(\mathrm{J} / \mathrm{KgK})$ & 1007,0 & & (m/seg) & 3,611 & & $(\mathrm{~m} / \mathrm{seg})$ & 3,4 & & (m/seg) & 8,7 \\
\hline Cond. Ter & $(\mathrm{W} / \mathrm{mK})$ & 0,0 & EV & & & EV & & & EV & & \\
\hline Dif. Termi & (m2/seg2) & 0,0 & Diametro & $(\mathrm{m})$ & 11,100 & Diametro & (m) & 11,1 & Diametro & $(\mathrm{m})$ & 11,1 \\
\hline Viscosidad & $\left(\mathrm{Kg} / \mathrm{m}^{*}\right.$ seg & 0,0 & $\mathrm{H}$ & $(\mathrm{m})$ & 3,600 & $\mathrm{H}$ & (m) & 3,6 & $\mathrm{H}$ & (m) & 3,6 \\
\hline Viscosidad & (m2/seg) & 0,0 & & & & ST & (m) & 19,0 & ST & $(\mathrm{m})$ & 19,0 \\
\hline $\mathrm{Pr}$ & $(-)$ & 0,7 & & & & SD & (m) & 21,2 & SL & $(\mathrm{m})$ & 19,0 \\
\hline Ra & $(-)$ & & & & & SL & (m) & 19,0 & & & \\
\hline $\operatorname{Re}$ & $(-)$ & 2566721 & & & & & & & & & \\
\hline $\mathrm{Nu}_{\text {laminar }}$ & $(-)$ & 258,9 & FALSO & $h_{\text {laminar me }}$ & 1,834 & FALSO & $h_{\text {laminar me }}$ & 1,8 & FALSO & $h_{\text {laminar me }}$ & 2,8 \\
\hline $\mathrm{Nu}_{\text {turbulent }}$ & $(-)$ & 4468,7 & URBULEN & $h_{\text {turbu }}$ & 31,653 & URBULEN & $h_{\text {turbul }}$ & 25,0 & JRBULENT & $h_{\text {turbulento }}$ & 55,8 \\
\hline
\end{tabular}

Tabla 2. Valores semi-experimentales de conductancias superficiales exteriores (he) para EV expuestas al viento en configuraciones aisladas, en conjuntos de EV escalonados y en conjuntos de EV alineados. Fuente: Elaboración de la autora.

\section{DISCUSIÓN DE LOS RESULTADOS}

\section{EFECTO DEL VIENTO SOBRE LA DISTRIBUCIÓN DE LAS EV}

Ante todo, se considera aquí tres tipos de ubicaciones posibles de la EV: aisladas o primeras EV, sobre las cuales el viento impacta a su velocidad original, y dos tipos de configuraciones en conjunto. Estas pueden ser alineadas o escalonadas, siendo las primeras aquellas EV ordenadas en hileras, mientras las escalonadas presentan irrupción de los corredores por los que transita el viento. En estas configuraciones en conjunto, la velocidad del viento se ve alterada, como se observa en la Tabla 2.
Se observa -también en la Tabla 2-, en función de la distribución de las EV en el terreno, que el viento sufre una aceleración mayor en aquellas configuraciones en conjuntos alineados y una leve disminución de su velocidad en conjuntos escalonados, respecto de la dirección predominante de las brisas. El viento impacta en su velocidad original sobre las primeras EV del conjunto. La turbulencia del viento $\left(h_{\text {turbulento }}\right)$ incrementa la velocidad del intercambio de energía entre el aire exterior y el vino. Este impacto es menor en las EV ubicadas escalonadamente frente al viento $\left(h_{\text {turbulento }}=25 \mathrm{~W} / \mathrm{m}^{2} \mathrm{~K}\right)$, y significativo en conjuntos alineados por cuyos corredores se acelera el flujo ( $h_{\text {turbulento }}=55,8 \mathrm{~W} / \mathrm{m}^{2} \mathrm{~K}$ ). Para el caso analizado, se toma la configuración alineada como si se considerara de una configuración escalonada, ya que el viento impacta en su impronta en ángulo de $45^{\circ}$ (ver Figura 2). 

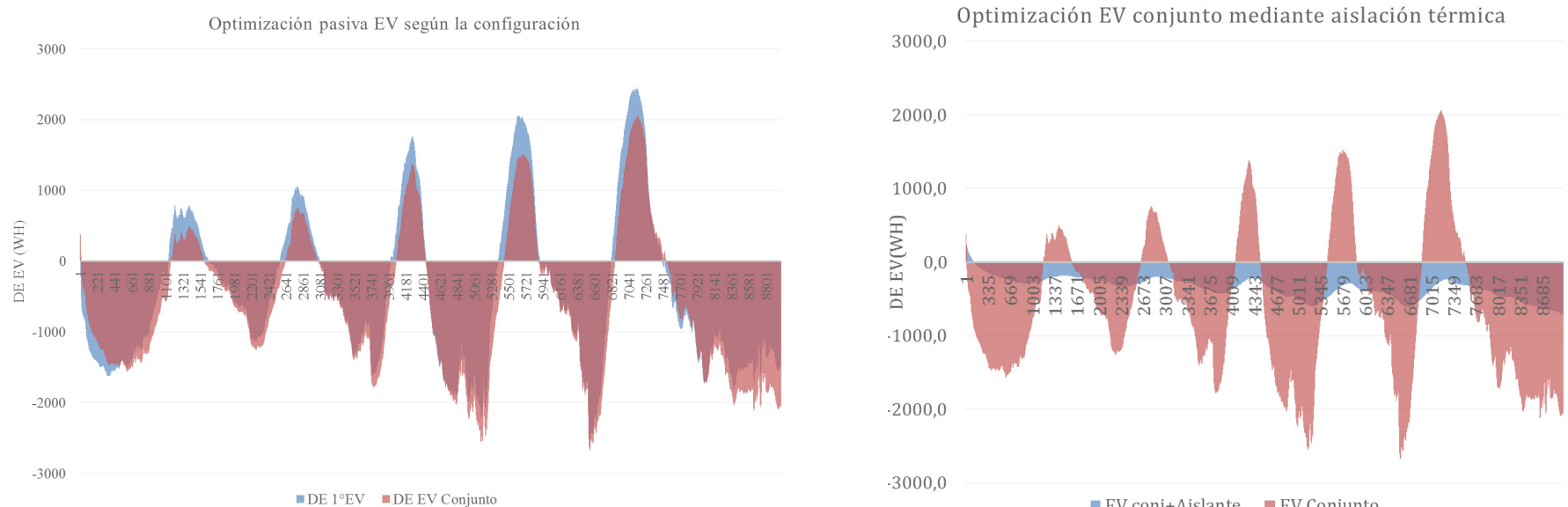

Figura 6. Resultados de la simulación de la variación de la demanda energética, según la ubicación de la EV.

Fuente: Elaboración de la autora.

Figura 7. Resultados de la simulación de la demanda energética, a partir de la optimización mediante la incorporación de aislación térmica a la EV.

Fuente: Elaboración de la autora.

\section{OPTIMIZACIÓN PASIVA DE LA EV}

Efecto de la optimización pasiva sobre la demanda energética $(D E)$, según la ubicación de la EV

En este punto, se simula la EV real modelada en diversas configuraciones dentro de la planta industrial, situada en conjunto cual banco de tubos (EV conjunto) y aislada (EV única). Luego, se comparan los resultados térmicos de la ubicación de las envolventes mencionadas en la configuración de la planta industrial. De esta forma, se observa que la selección de la EV a partir del efecto del viento sobre su estabilidad térmica tiene relativamente poca incidencia. Es decir, que a pesar de que existe un incremento de la velocidad del viento entre distintas configuraciones, este no incide de forma significativa en la DE. Sin embargo, como se observa en la Figura 6 es posible afirmar que la EV en la cual impactan las brisas en su velocidad original $\left(1^{\circ} \mathrm{EV}\right)$ presenta mayor amplitud térmica y menor estabilidad que el caso comparado, EV conjunto..

Efecto de la optimización pasiva sobre la $D E$, según la incorporación de aislación térmica en la EV a ambas configuraciones

Se toman, en este paso, ambas configuraciones de EV y se las optimiza mediante la incorporación superficial exterior de una capa de aislación térmica de espuma de poliuretano de 0,06 m de espesor (Cond. Térmica 0,029 $\mathrm{W} / \mathrm{mK}$ ), debajo de una capa de revoque para protección

mecánica, de similar espesor. Se evidencia, entonces, que los casos responden de forma semejante ${ }^{2}$, con una disminución significativa de su DE. Esta estrategia resulta, como se observa en la Figura 7, de una efectividad tan elevada -dadas las condiciones climáticas consideradas en este estudio- que permite alcanzar una demanda casi cero de energía en control térmico.

\section{Efecto de la optimización pasiva sobre la estabilidad} térmica del vino

Se observa aquí el desempeño térmico de la EV en cuatro situaciones: EV conjunto, EV conjunto+aislante, $1^{\circ} \mathrm{EV}$ y $1^{\circ} \mathrm{EV}$ +aislante. Se considera la estabilidad térmica del vino almacenado, en dirección espacial, es decir, desde el elemento muro hacia el punto más alejado del mismo, coincidente con el núcleo del vino. Por otra parte, se considera igualmente la estabilidad térmica del vino a lo largo de los ciclos diarios, en este caso, durante seis días corridos dentro del período estival.

\section{CONCLUSIONES}

Cuando se compara los resultados estadísticos de todos los casos simulados con los datos climáticos de temperatura exterior, se advierte que, si bien todos los casos de EV analizados superan los valores medios estadísticos de conservación del vino en verano $\left(22,2^{\circ} \mathrm{C}\right)$, se caracterizan por una inercia térmica relevante. Esto significa que, si el 
ESTABILIDAD TÉRMICA INTERIOR EN EL ESPACIO

$\square$ EV conjunto $\square$ EV conjunto+aislante $\square 1^{\circ} \mathrm{EV} \square 1^{\circ} \mathrm{EV}$ +aislante

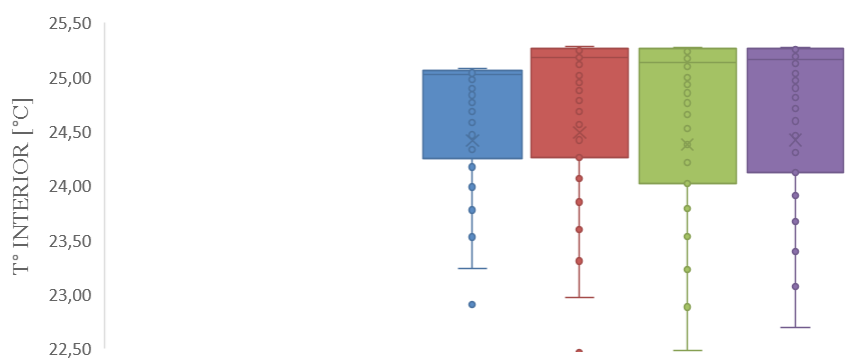

ESTABILIDAD TERMICA INTERIOR EN EL TIEMPO

$\square$ EV conjunto $\square$ EV conjunto+aislante $\square 1^{\circ} \mathrm{EV} \square 1^{\circ} \mathrm{EV}$ +aislante
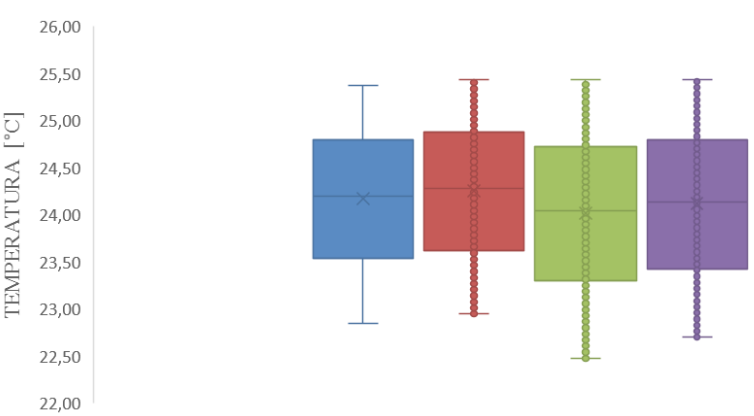

Figura 8. Gráfico de cajas para las temperaturas del vino almacenado en las envolventes simuladas y optimizadas. A la izquierda, la variación térmica en función del espacio (profundidad de la vasija) y, a la derecha, la variación térmica en función del tiempo (seis días). Fuente: Elaboración de la autora.

Los resultados de la optimización de la EV de conservación del vino se analizan estadísticamente, señalando una dispersión térmica diferente para cada caso, desde el nodo en contacto con el elemento EV hacia el núcleo del vino. Se observa en la Figura 8 que esta heterogeneidad térmica en el volumen de vino es mayor en el caso $1^{\circ} \mathrm{EV}$ con $+/-3,26 \mathrm{~K}$ y menor para EV conjunto escalonado, con +/- 1,8 K. Ello muestra que el vino varía térmicamente con cierta homogeneidad en configuraciones de planta industrial tipo conjunto escalonado, o sea, que tiene mayor estabilidad térmica en esta tipología que en el resto de los casos considerados. En este caso, la temperatura superficial interior media es más baja, dado que la línea de tendencia de la temperatura exterior durante el período de medición es descendente y la EV no está aislada. Los otros tres casos señalan una reducción del impacto de la onda térmica exterior. Si bien la temperatura media del vino es más cálida en la superficie en contacto con la envolvente, a medida que los nodos se distancian de esta, se observa el gran potencial de conservación de temperaturas más bajas, en todos los casos considerados de EV.

producto almacenado es pre-enfriado, el mismo conservará su temperatura por al menos un período de una semana, durante la cual se prescindirá del empleo de recursos termoenergéticos para suplir esta demanda. En dicho período la industria se ahorra energía eléctrica, garantizándose simultáneamente la estabilidad térmica del producto. El empleo de esta tipología de EV para la conservación del vino permite una eficiente gestión energética en las bodegas de la zona de análisis. El proceso puede ser complementado mediante recursos geotérmicos -cuya temperatura en la zona se mantiene en $\operatorname{los} 18^{\circ} \mathrm{C}$ - sin tratar, o enfriado mecánicamente, dependiendo de los márgenes térmicos demandados por la calidad del producto.

Si se compara, asimismo, los resultados de la simulación con los datos registrados para el caso real, se observa que presentan la misma performance energética, con gran estabilidad térmica del producto y mínima asistencia en control de temperatura. Ahora bien, si se compara, además de los anteriores, otros valores reales de una tipología industrial distinta, como los tanques de acero inoxidable, se pueden observar las diferencias. El tanque de acero se asemeja más en su desempeño térmico a la sinusoide de la temperatura exterior del clima que a los casos representativos reales y simulados de la EV de hormigón. El empleo de los tanques de acero inoxidable o metal en la industria parece no favorecer al uso racional y gestión de la energía. A pesar de encontrarse en el interior de una bodega, el estado térmico del vino almacenado en el tanque presenta oscilaciones casi idénticas a las del aire exterior.
En este trabajo se ha desarrollado una herramienta para el pre-diagnóstico energético de envolventes de tipo industrial, cuya aplicación es extensiva a agroindustrias regionales como las olivícolas, cerveceras, lecheras y todas aquellas en donde están implicadas grandes superficies de envolventes expuestas a la intemperie, temperaturas operativas y productos de valor agregado. Esta herramienta también puede aplicarse a envolventes de vinificación más comercialmente difundidas, como tanques metálicos, de PVC o acero inoxidable. Además, es posible complementar la herramienta con un modelo de fermentación alcohólica, integrando una planilla de ingreso de datos específicos (cantidad de azúcar, dióxido de carbono, densidad, etc.) con las expresiones correspondientes a los modelos cinéticos. Otra importancia del modelado es que refleja los cambios en la energía interna del producto guardado, cuya estabilidad térmica es la base de la preservación del mismo, particularmente, en las instancias posteriores a la fermentación, como lo constituyen la estabilización y guarda del producto.

El uso racional de la energía en la industria atiende principalmente a la calificación energética de los sistemas mecánicos porque es este el parámetro de eficiencia energética impuesto y difundido en el mundo desarrollado. Por su parte, la sustentabilidad edilicia se aplica sobre todo a edificios comerciales y residenciales. De esta manera, la eficiencia energética en las envolventes utilizadas en las industrias se caracteriza por escasas soluciones específicas. La definición de prototipos de envolventes eficientes desde 


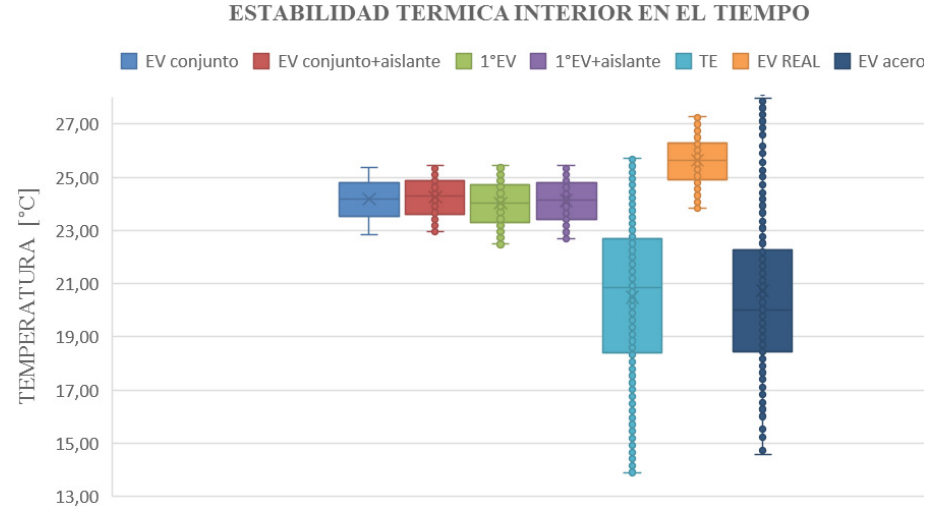

Figura 9. Gráfico de cajas para las temperaturas del vino almacenado en las envolventes simuladas y optimizadas y temperatura exterior. Fuente: Elaboración de la autora.

el uso racional de la energía es una tarea en desarrollo en numerosos trabajos publicados. Los resultados obtenidos en esta investigación siembran un pequeño aporte a los avances en materia de uso racional de la energía para la calificación, certificación y gestión energética de las industrias, en el contexto climático analizado. Se ha demostrado cuantitativamente y de manera comparativa el desempeño de la envolvente representativa en la conservación del vino, frente a una envolvente de tipo comercial, como el hegemónico y ampliamente utilizado tanque de acero inoxidable. Podría decirse, en definitiva, que, para el contexto climático analizado, existe un prototipo de envolvente industrial eficiente para la conservación del vino. Este tipo de envolventes, definidas por su gran poder de inercia térmica, constituye la tipología industrial que más se aproxima al concepto de Net Zero Buildings aplicado al contexto de las envolventes industriales.

\section{AGRADECIMIENTO}

Los autores agradecen a la empresa Vitícola Cuyo S.A. por proveer las instalaciones industriales para llevar a cabo el monitoreo térmico. Se agradece al Instituto Regional de Planeamiento y Hábitat (IRPHa) por proveer los instrumentos utilizados para realizar las mediciones. Se agradece, particularmente, al Consejo Nacional de Investigaciones Científicas y Tecnológicas (CONICET) por financiar este proyecto enmarcado la investigación Posdoctoral (2014/2016) de la Dra.-Arq. Ramos Sanz, Alba I.

\section{LISTADO DE SÍMBOLOS}

\section{$A A$ área $\left[\mathrm{m}^{2}\right]$}

oco difusividad térmica $\left[\mathrm{m}^{2} / \mathrm{seg}\right]$

$\Delta t \Delta t$ espaciamiento temporal [seg]

$\Delta x \Delta x$ espaciamiento espacial $[\mathrm{m}]$

$c_{p} c_{p}$ capacidad térmica específica $\left[\mathrm{J} / \mathrm{Kg}^{\circ} \mathrm{C}\right]$

$\rho \rho$ densidad $\left[\mathrm{Kg} / \mathrm{m}^{3}\right]$ $h$ coeficiente superficial de transferencia de calor $\left[\mathrm{Wh} / \mathrm{m}^{2 \circ} \mathrm{C}\right]$

$\lambda \lambda$ conductividad térmica del fluido $\left[\mathrm{Wh} / \mathrm{m}^{\circ} \mathrm{C}\right]$

L longitud [m]

M cantidad de nodos[u]

$\tau \tau$ número de Fourier [a]

$T_{n}^{\mathrm{l}} T_{n}^{\mathrm{t}}$ nodos $[\mathrm{u}]$

EV envolvente de vinificación

\section{REFERENCIAS BIBLIOGRÁFICAS}

AUSTRALIAN WINE RESEARCH INSTITUTE .Winery Energy Saver Toolkit [en línea], 2012. [Consultado 20 julio 2017]. Disponible en: http://www.winesa.asn.au

BRITISH COLUMBIA WINE GRAPE COUNCIL. Sustainable Winegrowing Program [en línea], 2011. [Consultado 20 julio 2017]. Disponible en: http://bcwgc.org

CALIFORNIA ENERGY COMMISSION. Best Winery [en línea], 2005. [Consultado 20 julio 2017]. Disponible en: www.energy. ca.gov

INSTITUTO NACIONAL DE VITIVINICULTURA, INV. [en línea]. 2008. [Consultado 20 julio 2017]. Disponible en: http://www.inv. gov.ar

INSTITUTO ARGENTINO DE NORMALIZACIÓN IRAM11603. Clasificación Bioambiental de la República Argentina. Buenos Aires, Argentina, 1996.

INSTITUTO ARGENTINO DE NORMALIZACIÓN IRAM/ISO50001. Gestión de la Energía en la Industria. Buenos Aires, Argentina, 2016.

OBSERVATORIO ECONÓMICO SOCIAL UNR [en línea]. [Consultado octubre 2017]. Disponible en: http://www. observatorio.unr.edu.ar/llego-la-factura-de-la-luz/

OREGLIA, Francisco. Enología Teórico-práctica. Ediciones Instituto Seliciano de Artes Gráficas, Buenos Aires, 1978.

RAMOS SANZ, Alba. El Confort del Vino en la Envolvente Industrial. Estudio de casos de vasijas vinarias de Bodegas argentinas. Editorial Publicia, Saarbrücken, Alemania, 2014.

RAMOS SANZ, Alba. Reducción de la demanda térmica originada en la vinificación en bodegas localizadas en zona de clima árido, mediante modelado térmico semi-empírico. Revista Hábitat Sustentable, Chile, 2015, vol. 5, n², pp. 6-19. 